\title{
Simultaneous Occurrence of Melanotic Macule and Melanoma in the Oral Cavity: A Case Report
}

\author{
Su-Hyun Kim, Jin-Seok Byun, Jae-Kwang Jung, Jae-Kap Choi \\ Department of Oral Medicine, School of Dentistry, Kyungpook National University, Daegu, Korea
}

Received September 7, 2020

Revised September 14, 2020 Accepted September 15, 2020

Correspondence to:

Jae-Kap Choi

Department of Oral Medicine, School of Dentistry, Kyungpook National University,

2175 Dalgubeol-daero, Jung-gu, Daegu 41940, Korea

Tel: $+82-53-600-7321$

Fax: +82-53-426-2195

E-mail: jhchoi@knu.ac.kr

https://orcid.org/0000-0001-6773-7507
While oral melanotic macule is regarded as the most frequent oral pigmented lesion without any harmful symptom, oral melanoma is a very rare and life-threatening disease among oral pigmented lesions. Oral melanotic macule has previously been described as benign, with no malignant potential. However, a few case reports have raised the question of malignant potential of oral melanotic macule. In this case report, we present a case of coexistence of oral melanotic macule and oral melanoma. A 66-year-old man with a chief complaint of black pigmentation on gingiva showed the lesion spread out on the right palatal gingiva, the right maxillary buccal gingiva and the right buccal mucosa, appearing to merge with one another. Biopsies were performed on the right palatal gingiva and the right buccal mucosa. While the lesion on the right palatal gingiva was diagnosed as an oral melanotic macule, the other lesion on the right buccal mucosa was diagnosed as an oral melanoma. The present case implied the possibility of malignant potential of oral melanotic macule. Therefore, oral melanotic macule needs careful periodic observation for early detection and prompt treatment of the transformed oral melanoma.

Key Words: Melanoma; Melanosis; Precancerous conditions

\section{INTRODUCTION}

Oral melanotic macule and oral melanoma are pigmented lesions of melanocytic origin that are found in the oral cavity. Oral melanotic macule is most prevalent, accounting for about 86\% of all melanocytic lesions [1]. Oral melanotic macules usually appear as asymptomatic and well-demarcated lesions with a diameter $<1 \mathrm{~cm}$ that mainly occur on the lower lip [2]. It is predominantly affecting in women over the fifth decade of life [3].

Unlike oral melanotic macule, oral melanoma is very rare, accounting for $0.2 \%$ to $8 \%$ of all melanomas [4]. Oral melanoma is most common on the hard palate that takes up about $40 \%$ of all cases, followed by maxillary gingiva that accounted for one third of all cases $[5,6]$. Oral melanoma occurs preponderantly in men aged 40 to 70 years
[4,7]. Clinically, oral melanoma also presents as a rapidly growing asymptomatic flat-pigmented lesion [8]. As these two conditions are very similar in clinical appearance, it is not easy to differentiate between the two lesions with clinical examination alone. Histological examination is strongly recommended for accurate differential diagnosis, especially in suspicious lesions. While oral melanoma is histologically characterized by the proliferation of atypical melanocytes along the junction between the epithelial and connective tissues, as well as within the connective tissue; oral melanotic macule is characterized by the increased melanin production in the basal cell layer of the epithelium without any melanocyte proliferation [8]. Unlike oral melanoma, it is generally considered that oral melanotic macule does not require surgical intervention. However, the possible malignant potential was cautiously suggested on oral melanotic

Copyright (c) 2020 Korean Academy of Orofacial Pain and Oral Medicine. All rights reserved.

(c) This is an open-access article distributed under the terms of the Creative Commons Attribution Non-Commercial License (http://creativecommons.org/licenses/by-nc/4.0/), which permits unrestricted non-commercial use, distribution, and reproduction in any medium, provided the original work is properly cited. 
macule in some case reports [9-15].

This manuscript reports the case of a 66-year-old man with the simultaneous occurrence of oral melanotic macule and oral melanoma.

\section{CASE REPORT}

A 66-year-old man with a chief complaint of non-painful black gums was referred to the Department of Oral Medicine of the Kyungpook National University Dental Hospital from a local dental clinic. He discovered the lesion accidentally 4 days before the visit. His medical history revealed that he was diagnosed with cardiac arrhythmia 3 years previously, and had taken $1.25 \mathrm{mg}$ of bisoprolol fumarate per day for 1 year.

Clinical examination of the oral cavity revealed flat brown-black pigmented lesions arising from the right maxillary buccal gingiva, extending from the right maxillary second molar to the left maxillary lateral incisor region, the right palatal gingiva, and the right buccal mucosa. The lesions on the right buccal mucosa were broadly spread and fused with one another, connected to the right palatal lesion (Fig. 1). There was no pain on palpation, and the lesion was not blanching on compression. No significant findings were observed on panoramic radiograph. The pigmented lesions were tentatively diagnosed as oral melanotic macule; however, oral melanoma needed to be ruled out.
Considering the large size of the lesions, incisional biopsies were conducted on two areas, the right palatal gingiva and the right buccal mucosa in order to rule out malignancy. Histopathologic evaluation of the right palatal gingiva revealed melanin deposition in the basal layer without any increase in melanocyte proliferation (Fig. 2). Based on these histological findings, the lesion on the right palatal gingiva was diagnosed as an oral melanotic macule. Meanwhile, histopathologic examination of the right buccal mucosa showed prominent proliferation of abnormal melanocytes, loss of the rete ridge, hyperchromatic nuclei, and nesting formation with abnormal melanocytes (Fig. 2). Based on these histological results, the lesion on the right buccal mucosa was diagnosed as an oral melanoma. Immunohistochemical staining for S100, HMB-45, and Melan A were also performed to confirm the histological diagnoses, and stains for S100 and HMB-45 were positive on both the areas that supported the diagnosis of oral melanoma of the right buccal mucosa. He was immediately referred to the Department of Oral and Maxillofacial Surgery at the Kyungpook National University Dental Hospital for surgical treatment. Further examinations, including computed tomography (CT), neck and chest $\mathrm{CT}$, positron emission tomography-CT, and magnetic resonance imaging, were performed and showed no evidence of metastases to other sites. The complications of surgical excision are considered too big to be acceptable by the patient; therefore,
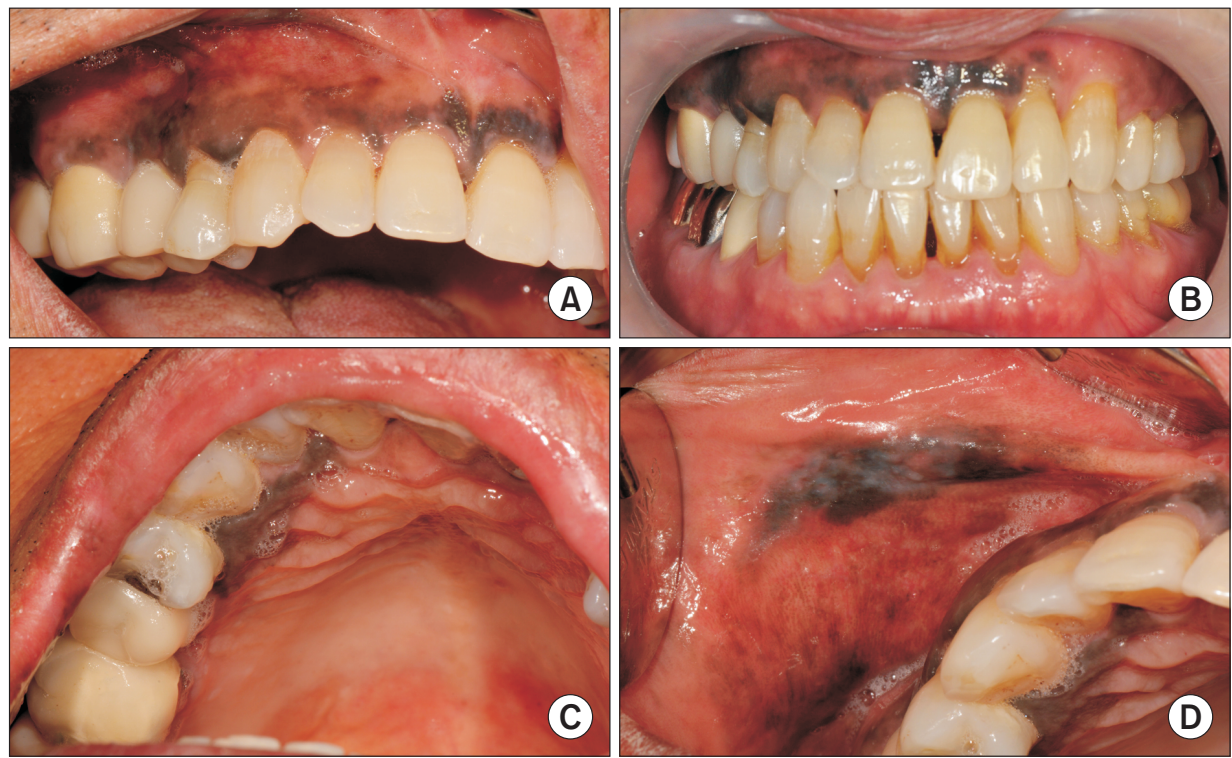

Fig. 1. Clinical photographs showing a pigmented lesion. (A) A brownblack pigmented lesion on the right buccal attached gingiva. (B) The lesion is extending from the right maxillary second molar to the left maxillary lateral incisor area. (C) The palatal aspect of the lesion. (D) The flat and non-ulcerated lesion of the right buccal mucosa. 

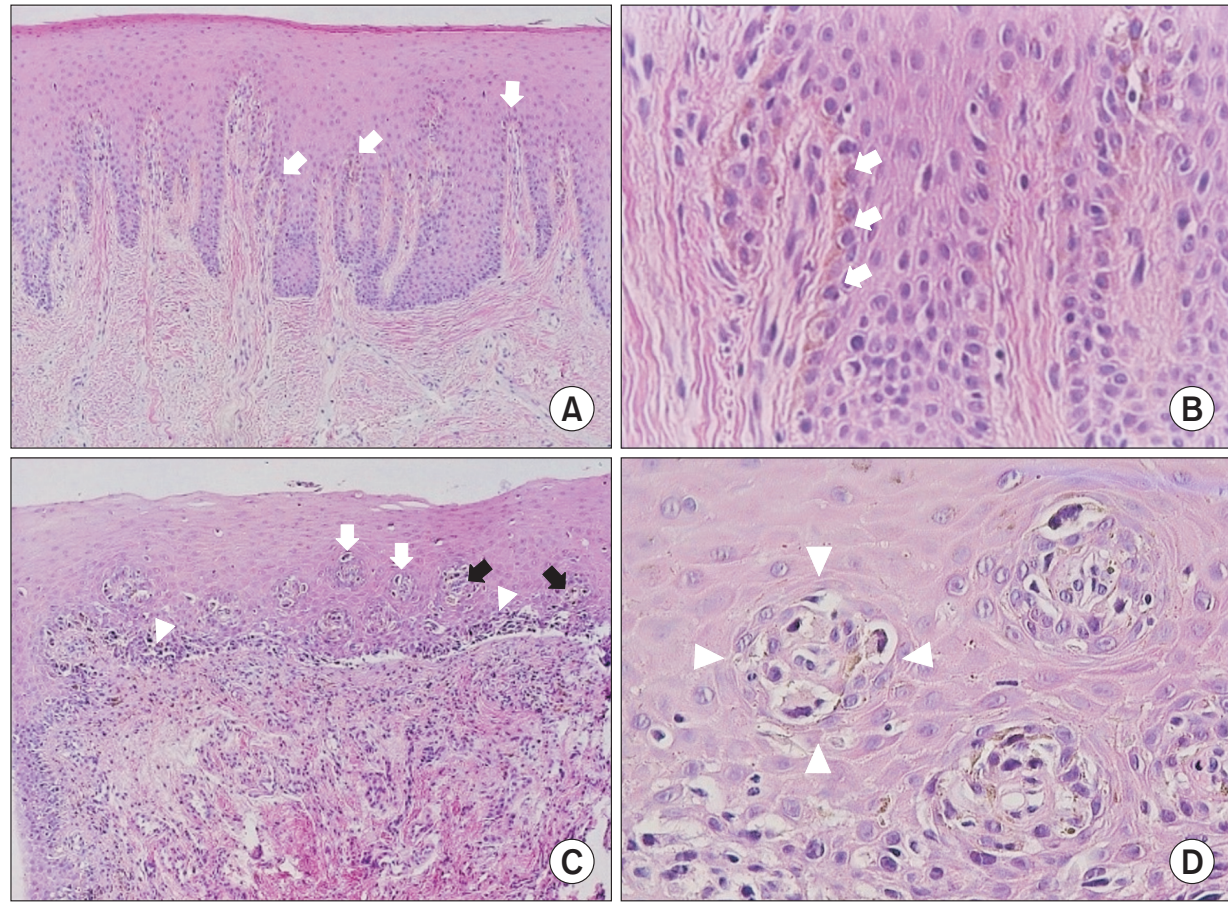

Fig. 2. Histopathological findings. $\mathrm{H} \& \mathrm{E}$ staining. (A) On the right palatal gingiva, increased melanin deposition along the basal cell layer (arrows) (magnification, $\times 100)$. (B) No increase in atypical melanocytes on the right palatal gingiva (arrows) (magnification, $\times 400$ ). (C) On the right buccal mucosa, increased the atypical melanocytes (white arrows), nuclear pleomorphism (black arrows), hyperchromatism and loss of rete ridges (arrow heads) are noted (magnification, $\times 100$ ). (D) Prominent nesting of atypical melanocytes on the right buccal mucosa (arrow heads) (magnification, $\times 400)$.
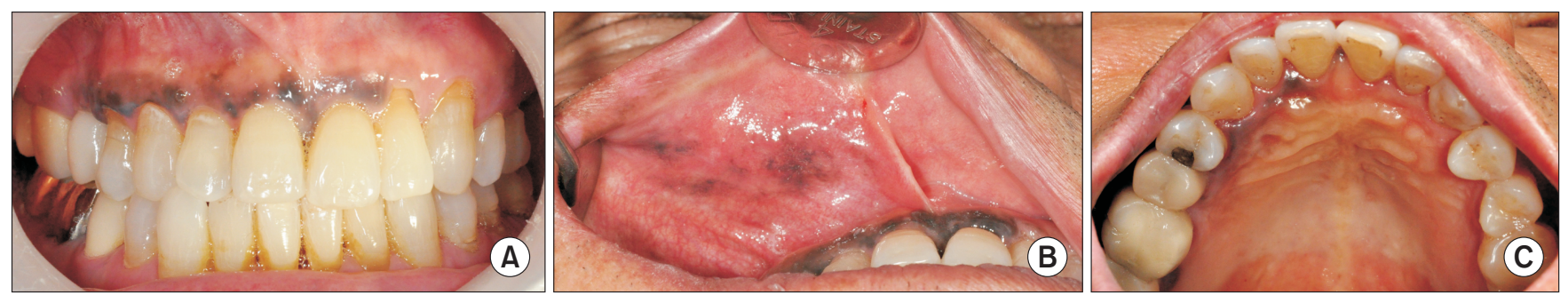

Fig. 3. After 1 year of the treatment, the size of the lesion has been reduced and the color of the lesion has become pale. The diminished lesion is observed on (A) the right buccal gingiva, $(B)$ the right buccal mucosa, and $(C)$ the right maxillary palate.

immunochemotherapy was performed at the Department of Hemato-Oncology at the Kyungpook National University Hospital. The patient showed improvement in the treatment. The size of the lesion has been gradually reduced and the color of the lesion has become lighter over the past year (Fig. 3). The patient has continued immunochemotherapy up to the present time.

\section{DISCUSSION}

Melanoma is a malignant neoplasm displaying the unrestrained growth of atypical melanocytes. The etiology of melanoma remains unclear. Melanocytes are neuroectodermal derivatives known to migrate to the skin, retina, uveal tract, and other ectodermally derived mucosa. Melanocytes also migrate at a relatively low frequency to the endodermally derived mucosa, such as the nasopharynx, larynx, esophagus, and oral cavity. Thus, melanoma can occasionally occur on mucosal surfaces although it usually develops on skin $[16,17]$. The most prevalent sites of mucosal melanomas were the head and neck region (55.4\%), followed by the anal/rectal region (23.8\%), as per a previous study in the United States from 1985 to 1994 [18]. The nasal cavity, paranasal sinuses, and oral cavity are the most common areas of mucosal melanoma of the head and neck region, though oral melanoma is very rare, accounting for $0.5 \%$ of all oral malignancies [19,20].

While the etiology of oral melanoma is not clearly established, it is generally known to arise de novo. However, as per a previous study, about 30\% of 93 oral melanoma 
patients had already exhibited the pigmented lesion from several months to years before their diagnoses [21]. Other studies have also reported that the presence of oral pigmented lesion proceeded the diagnosis of oral melanoma in $35 \%$ to $37 \%$ of the patients $[4,5,22]$. However, these studies were limited by a lack of histopathologic information on the pre-existed pigmentations. To the best of our knowledge, only 8 cases of oral melanoma describing the detailed histopathologic features of the pre-existed pigmented lesion have been published in English. A summary of these 8 cases is presented in Table 1 [9-15]. The pigmentations in these cases were initially diagnosed as follows: benign gingival melanosis, melanocytic dysplasia, oral melanotic macule, oral melanosis, premalignant melanosis, melanocytic hyperplasia and oral melanoacanthoma. In 4 of the 8 cases, a histological diagnosis of oral melanotic macule was initially established. However, the follow-up biopsy performed on the same area showed that the oral melanotic macule was spontaneously transformed into oral melanoma. Oral melanotic macule was generally known as a benign pigmented lesion without malignant potential [23-26]. However, serial biopsies performed for the 4 patients suggested the malignant potential of the oral melanotic macule. Unlike these previous cases, the biopsy in the present case was simultaneously performed on two regions, the right palate and the right buccal mucosa. The lesion is widespread on the right side of the oral cavity and is connected to one another; therefore, we could not confirm that two regions where the biopsies were taken were completely independent. Thus, whether the two lesions developed independently or the oral melanotic macule was transformed to oral melanoma could not be demonstrated. Furthermore, the possibility of malignant potential of this oral melanotic macule should not be excluded.

The asymmetry, border irregularity, color variegation, diameter, and elevation or evolution (ABCDE) criteria are useful for the clinical assessment of cutaneous melanoma [27]. However, mucous membranes have a structure that is different from that of the skin; this makes it difficult to apply the $\mathrm{ABCDE}$ criteria in the clinical assessment of oral melanoma. In order to establish the exact diagnosis of oral melanoma, the combination of hematoxylin and eosin (H\&E) staining and immunohistochemical staining is considered the most reliable diagnostic method for oral melanoma. In particular, several diagnostic markers including HMB-45, S100, and Melan A, are widely used for evaluating the immunohistochemical profiles of oral melanoma. The histologic features of oral melanoma are very similar to those of other tumors such as carcinoma, sarcoma, plasmacytoma, lymphoma, and germ cell tumors. The immunohistochemical staining is used to distinguish poorly differentiated melanomas from these other tumors. However, the markers are not used to distinguish oral melanoma from oral melanotic macule, since these markers are positively expressed in both oral melanoma and oral melanotic macule. Therefore, a diagnosis of pigmented lesion is mainly based on biopsy with $H \& E$ staining, and in some cases, immunohistochemical staining supplements a diagnosis with H\&E staining [28]. Moreover, when the size of the pigmented lesion is relatively large, clinicians are recommended to perform biopsies on several areas. In this case, the lesion was large; therefore, biopsies were performed at 2 different sites, the right palate and the right buccal mucosa. The right palate was considered as a biopsy site, since it is a site where oral melanoma is most prevalent. The right buccal mucosa was also selected as a biopsy site due to its irregular shape and color variegation. Biopsy was not performed on the right maxillary buccal gingiva due to concerns about side effect of gingival recession. As a result, oral melanoma was diagnosed in the right buccal mucosa, which is not the predominant site of oral melanoma. If biopsy was only performed on the palatal site, the diagnosis of oral melanoma would have been missed, potentially leading to poor prognosis owing to late detection of oral melanoma.

Oral melanotic macule has long been considered a benign lesion without malignant potential; thus, follow-up has been somewhat neglected. However, considering the previous and present cases, the possible malignant potential of oral melanotic macule should be considered, and careful follow-up should be performed. If diagnosis is delayed due to ignorance, the prognosis is very poor. Delayed detection is considered to be a reason for the worse prognosis of oral melanoma with a low 5-year survival rate of 15\% to $40 \%$ [29-32]. Further, oral melanotic macule is the most common pigmented lesion of the oral cavity; therefore, it is clinically significant to consider the possibility of malignant 


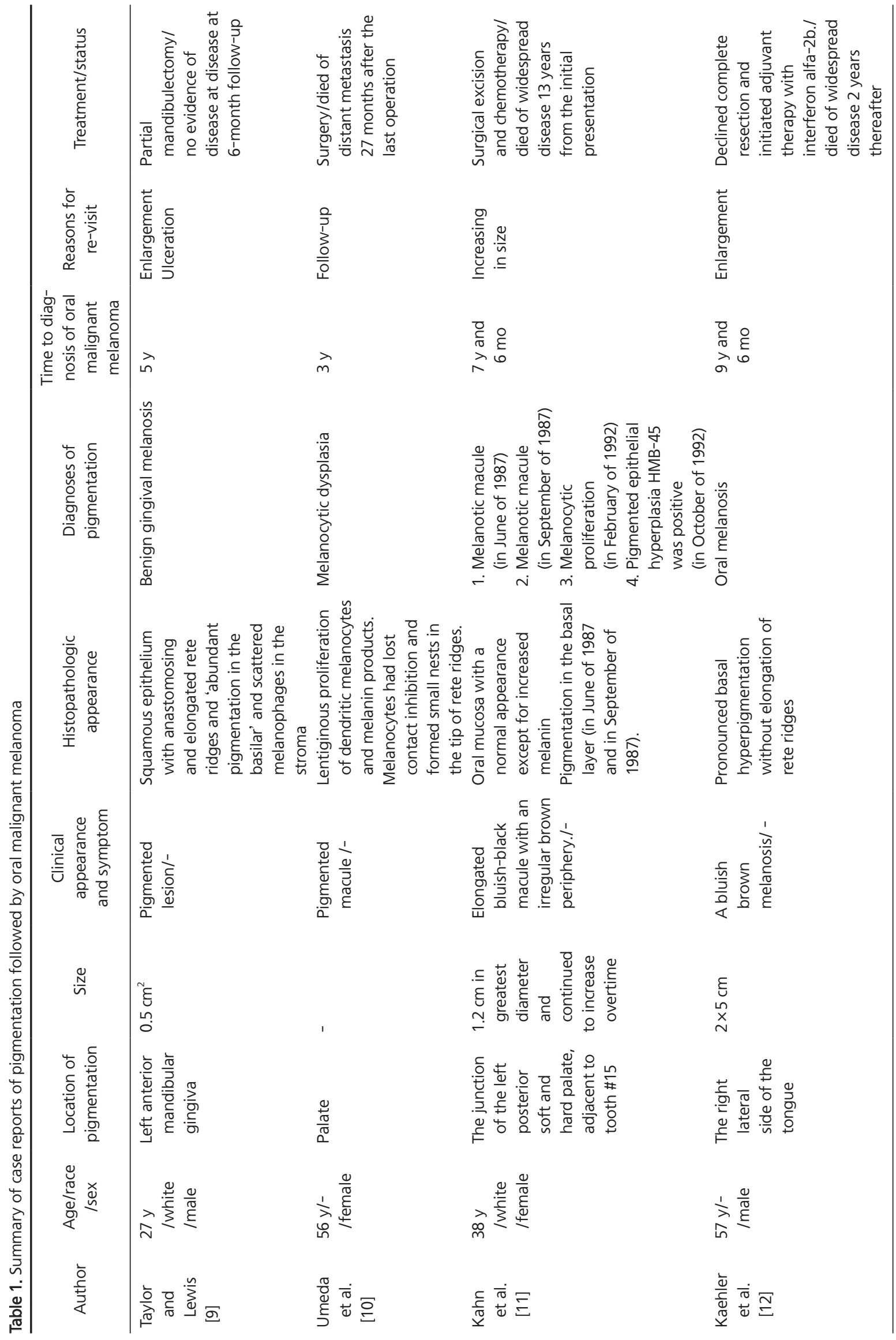




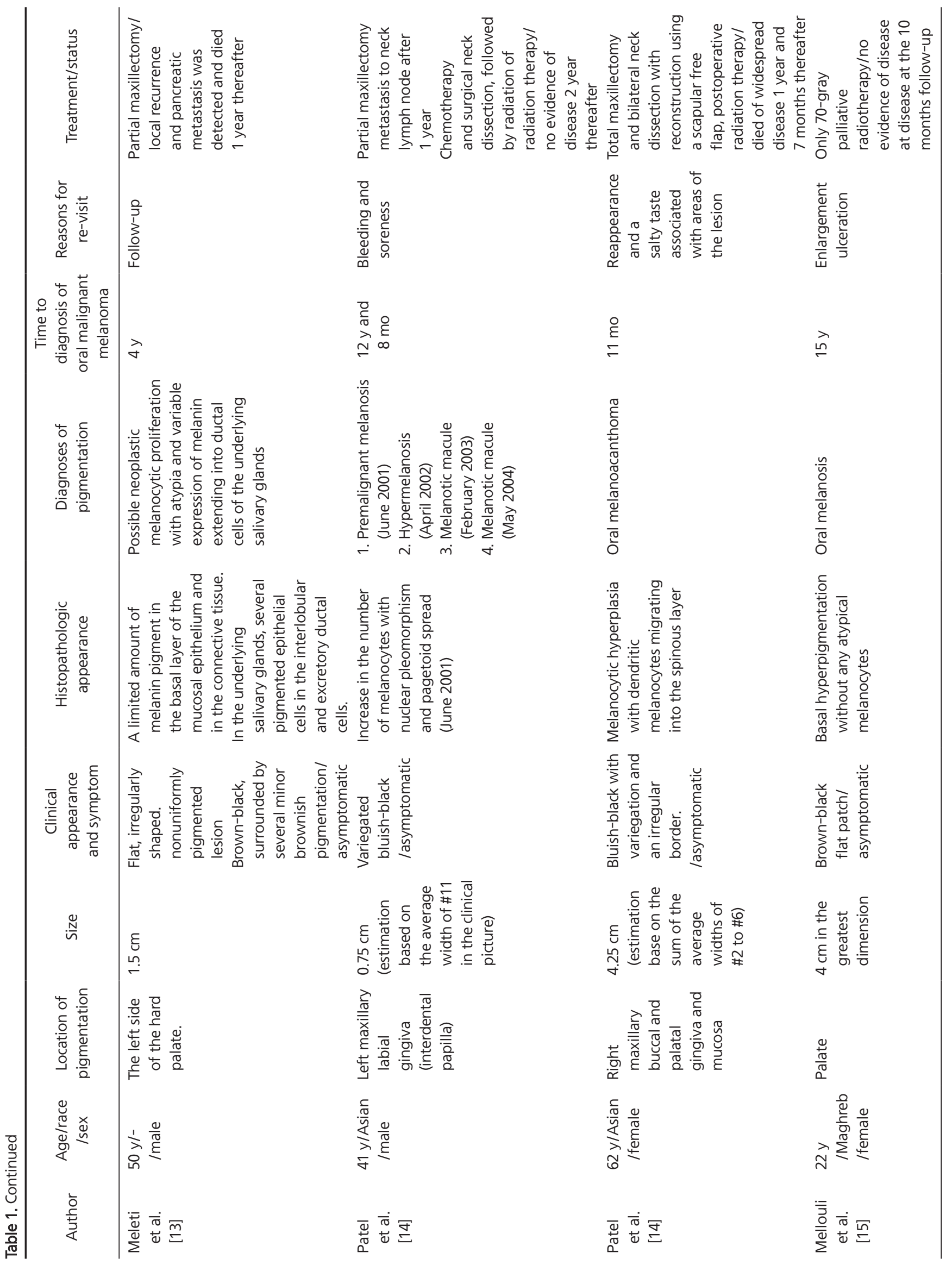


potential of oral melanotic macule. Oral melanoma, unlike oral melanotic macule, is likely to change rapidly over a period of time. Therefore, it is critical to carefully monitor the change in the patterns via periodic observation to facilitate early detection and, ultimately, better prognosis of oral melanoma. As there were only a few cases indicating the possible malignant potential of oral melanotic macule, further detailed study is necessary for determining the malignant potential and the underling pathological mechanism of oral melanotic macule.

\section{CONFLICT OF INTEREST}

No potential conflict of interest relevant to this article was reported.

\section{ORCID}

\author{
Su-Hyun Kim \\ https://orcid.org/0000-0002-1410-3671 \\ Jin-Seok Byun \\ https://orcid.org/0000-0002-6182-1238 \\ Jae-Kwang Jung \\ https://orcid.org/0000-0003-3099-8097 \\ Jae Kap Choi \\ https://orcid.org/0000-0001-6773-7507
}

\section{REFERENCES}

1. Buchner A, Merrell PW, Carpenter WM. Relative frequency of solitary melanocytic lesions of the oral mucosa. J Oral Pathol Med 2004;33:550-557.

2. Weathers DR, Corio RL, Crawford BE, Giansanti JS, Page LR. The labial melanotic macule. Oral Surg Oral Med Oral Pathol 1976;42:196-205.

3. Shen ZY, Liu W, Bao ZX, Zhou ZT, Wang LZ. Oral melanotic macule and primary oral malignant melanoma: epidemiology, location involved, and clinical implications. Oral Surg Oral Med Oral Pathol Oral Radiol Endod 2011;112:e21-e25.

4. Rapini RP, Golitz LE, Greer RO Jr, Krekorian EA, Poulson T. Primary malignant melanoma of the oral cavity. A review of 177 cases. Cancer 1985;55:1543-1551.

5. Takagi M, Ishikawa G, Mori W. Primary malignant melanoma of the oral cavity in Japan. With special reference to mucosal melanosis. Cancer 1974;34:358-370.

6. Hicks MJ, Flaitz CM. Oral mucosal melanoma: epidemiology and pathobiology. Oral Oncol 2000;36:152-169.
7. Macintyre DR, Briggs JC. Primary oral malignant melanoma. Int J Oral Surg 1984;13:160-165.

8. Kauzman A, Pavone M, Blanas N, Bradley G. Pigmented lesions of the oral cavity: review, differential diagnosis, and case presentations. J Can Dent Assoc 2004;70:682-683.

9. Taylor CO, Lewis JS. Histologically documented transformation of benign oral melanosis into malignant melanoma: a case report. J Oral Maxillofac Surg 1990;48:732-734.

10. Umeda M, Komatsubara H, Shibuya Y, Yokoo S, Komori T. Premalignant melanocytic dysplasia and malignant melanoma of the oral mucosa. Oral Oncol 2002;38:714-722.

11. Kahn MA, Weathers DR, Hoffman JG. Transformation of a benign oral pigmentation to primary oral melanoma. Oral Surg Oral Med Oral Pathol Oral Radiol Endod 2005;100:454-459.

12. Kaehler KC, Russo PA, Egberts F, Warnke PH, Cerroni L, Hauschild A. Metastatic melanoma of the tongue arising from oral melanosis. Arch Dermatol 2008;144:558-560.

13. Meleti M, Mooi WJ, van der Waal I. Melanotic pigmentation of palatal salivary glands as a possible precursor to malignant melanoma: report of an unusual case. J Oral Maxillofac Surg 2010;68:867-869.

14. Patel PB, Wright JM, Kang DR, Cheng YL. Longitudinal clinicopathologic data of the progression of oral mucosal melanomareport of 2 cases and literature review. Oral Surg Oral Med Oral Pathol Oral Radiol 2018;126:e21-e30.

15. Mellouli N, Sioud S, Garma M, Chokri A, Hamdi H, Selmi J. Oral malignant melanoma: History of malignant degeneration of a pigmented lesion. J Oral Med Oral Surg 2019;25:19.

16. Allen AC, Spitz S. Malignant melanoma; a clinicopathological analysis of the criteria for diagnosis and prognosis. Cancer 1953;6:1-45.

17. Femiano F, Lanza A, Buonaiuto C, Gombos F, Di Spirito F, Cirillo N. Oral malignant melanoma: a review of the literature. J Oral Pathol Med 2008;37:383-388.

18. Chang AE, Karnell LH, Menck HR. The National Cancer Data Base report on cutaneous and noncutaneous melanoma: a summary of 84,836 cases from the past decade. Cancer 1998;83:1664-1678.

19. Ascierto PA, Accorona R, Botti G, et al. Mucosal melanoma of the head and neck. Crit Rev Oncol Hematol 2017;112:136-152.

20. Ostman J, Anneroth G, Gustafsson H, Tavelin B. Malignant oral tumours in Sweden 1960-1989: an epidemiological study. Eur J Cancer B Oral Oncol 1995;31B:106-112.

21. Chaudhry AP, Hampel A, Gorlin RJ. Primary malignant melanoma of the oral cavity: a review of 105 cases. Cancer 1958;11:923928.

22. Liversedge RL. Oral malignant melanoma. Br J Oral Surg 1975; 13:40-55.

23. Kaugars GE, Heise AP, Riley WT, Abbey LM, Svirsky JA. Oral melanotic macules: a review of 353 cases. Oral Surg Oral Med Oral Pathol 1993;76:59-61.

24. Gupta G, Williams RE, Mackie RM. The labial melanotic macule: a review of 79 cases. Br J Dermatol 1997;136:772-775.

25. Buchner A, Hansen LS. Melanotic macule of the oral mucosa. A clinicopathologic study of 105 cases. Oral Surg Oral Med Oral Pathol 1979;48:244-249. 
26. Ho KK, Dervan P, O'Loughlin S, Powell FC. Labial melanotic macule: a clinical, histopathologic, and ultrastructural study. J Am Acad Dermatol 1993;28:33-39.

27. Thomas L, Tranchand P, Berard F, Secchi T, Colin C, Moulin G. Semiological value of ABCDE criteria in the diagnosis of cutaneous pigmented tumors. Dermatology 1998;197:11-17.

28. Buery RR, Siar CH, Katase N, et al. Clinico-pathological evaluation of oral melanotic macule, oral pigmented nevus and oral mucosal melanoma. J Hard Tissue Biol 2010;19:57-64.

29. Patrick RJ, Fenske NA, Messina JL. Primary mucosal melanoma.
J Am Acad Dermatol 2007;56:828-834.

30. Meleti M, Leemans CR, Mooi WJ, Vescovi P, van der Waal I. Oral malignant melanoma: a review of the literature. Oral Oncol 2007;43:116-121.

31. Tanaka N, Mimura M, Ogi K, Amagasa T. Primary malignant melanoma of the oral cavity: assessment of outcome from the clinical records of 35 patients. Int J Oral Maxillofac Surg 2004; 33:761-765.

32. Patel SG, Prasad ML, Escrig M, et al. Primary mucosal malignant melanoma of the head and neck. Head Neck 2002;24:247-257. 